\title{
Low-Cycle Fatigue Crack Growth in Ti-6242 at Elevated Temperature
}

\author{
Rebecka Brommesson ${ }^{1, a}$, Magnus Hörnqvist ${ }^{2, b}$, and Magnus Ekh ${ }^{3, c}$ \\ 1,3 Department of Applied Mechanics, Chalmers University of Technology, Göteborg, Sweden \\ ${ }^{2}$ Department of Applied Physics, Chalmers University of Technology, Göteborg, Sweden \\ ${ }^{2}$ ResearchCentre, GKN Aerospace Engine Systems, Trollhättan, Sweden \\ arebecka.brommesson@chalmers.se, bmagnus.hornqvist@gknaerospace.com, \\ 'magnus.ekh@chalmers.se
}

\begin{abstract}
Keywords: Low-Cycle Fatigue Crack Growth, Finite Element, Constitutive Model, Mean Stress Relaxation
\end{abstract}

\begin{abstract}
During low-cycle fatigue test with smooth bars the number of cycles to initiation is commonly defined from a measured relative drop in maximum load. This criterion cannot be directly related to the actual measure of interest - the crack length. By relating data from controlled crack growth tests under low-cycle fatigue conditions of a high strength Titanium alloy at $350^{\circ} \mathrm{C}$ and numerical simulation of these tests, it is shown that it is possible to determine the relationship between load drop and crack length, provided that care is taken to consider all relevant aspects of the materials stress-strain response.
\end{abstract}

\section{Introduction}

Traditional low-cycle fatigue (LCF) testing is performed using either smooth round bars or test specimens with notched geometries to resemble relevant features in components. During LCF tests microcracks are initiated relatively early in the fatigue life, and the majority of cycles are spent propagating the dominating crack to failure. For many applications, the number of cycles to failure $\left(N_{f}\right)$ is an inadequate measure since the aim is to determine when a macroscopic crack has formed, so that a subsequent crack propagation analysis may provide a more accurate estimation of the total life in a component of arbitrary geometry. The number of cycles to initiation $\left(N_{i}\right)$ is usually defined as the point where a given metric, which is either continuously evaluated during testing or examined postmortem, exceeds some pre-determined limit. In strain controlled testing of smooth specimens, the most common metric is the $X \%$ load drop criteria [1], where initiation is defined as the point where the maximum load has dropped below $\mathrm{X} \%$ of the saturated stable value (or in the case of continuously increasing or decreasing maximum stress the load drop compared to the extrapolated values of stress is used).

Although practical, the inherent drawback of the criterion is that it is not related to the size of the crack, which is the measure that is of actual interest. If the LCF data is to be used to directly assess the life of a component, the crack size at the point of initiation must be known and compared to the dimensions of the critical location in the component. If the definition of initiation results in large crack sizes before the load drop criterion is used, the data may give un-conservative life predictions for e.g. thin-walled components. Furthermore, to allow subsequent crack propagation analysis the crack size at the point of initiation must be known. It is therefore of interest to investigate how the drop in maximum load and crack length are related during LCF testing. In addition, for round bars where the crack initiation may occur randomly around the length and circumference of the gage section, the location of the extensometer relative to the point of initiation can play a dominating part in the correspondence between crack length and load drop.

In this work, we aim to demonstrate that a Chaboche-type of constitutive model can be used to assess the relationship between crack length and load drop during high-temperature LCF crack growth, provided that correct measures are taken to account for mean stress development. We also discuss the influence of extensometer location relative to the crack. 


\section{Experimental}

Materials and testing. The selected material is the forged high temperature $\alpha / \beta$ Ti-6Al-2Sn-4Zr-2Mo (-0.1Si) alloy (hereafter denoted Ti-6242), composition in weight percent, in bar form. Specimens of the surface flaw Kb-type [2] for fatigue crack growth testing were machined with the loading axis in the longitudinal direction. The specimens had a rectangular cross-section of $10.2 \times 4.3 \mathrm{~mm}$ in the $32 \mathrm{~mm}$ long gage section. A starter notch, centred on the wide face of the specimen, was machined using electric discharge machining (EDM). The specimens were instrumented for direct current potential drop (DCPD) measurements, with PD probes attached across the crack and at a location away from the cracked cross-section to provide a reference signal. Fatigue cracks of length 0.6-0.7 $\mathrm{mm}$ were generated in stress control at room temperature using a $10 \mathrm{~Hz}$ trapezoidal waveform with $R_{\sigma}=\sigma_{\min } / \sigma_{\max }=0$, at linear elastic fracture mechanics (LEFM) conditions. High strain fatigue crack growth tests were carried out at $350^{\circ} \mathrm{C}$, heated using a conventional resistance furnace and controlled by a thermo-couple attached to the specimen, under total strain control with the strain measured by a $12 \mathrm{~mm}$ gage length extensometer attached to the side face of the specimen, centred around the cracked cross-section. The applied total strain range, $\Delta \epsilon$, was $1.2 \%$ in all tests with $R_{\epsilon}=\epsilon_{\min } / \epsilon_{\max }=0$ and 0.6 (two tests at each condition) at a frequency of $0.5 \mathrm{~Hz}$. Maximum and minimum stress were recorded for every 10 cycles and full hysteresis loops were recorded at logarithmic intervals. The testing followed the ASTM standards $[3,4]$ in all applicable aspects except for the strain control and violation of LEFM conditions. The criterion for crack length was violated for all cracks lengths, and the ligament condition was violated for crack lengths above $1.5 \mathrm{~mm}$ and $1.3 \mathrm{~mm}$ for $R_{\epsilon}=0$ and 0.6 , respectively.

Modelling. The material behaviour of Ti-6242 is modelled using a rate independent plasticity model originally formulated in [5]. The first 500 cycles of the high strain fatigue crack growth experiments have been used for material parameter identification. For these cycles the influence of the crack is assumed to be small. Due to the high strain ratios the experiments show mean stress relaxation for the first 100 cycles to semi-stable nonzero values as can be observed in Fig. 2. For the material model used this can be captured by combining nonlinear and linear terms of kinematic hardening, cf. [6]. By this approach a poor fit with respect to experiments is obtained for the first cycle, where the model response overestimates the maximum stress. However, the material response closely follows experimental results for later cycles as can be seen in Fig. 1. A better model response with respect to experimental data could probably be obtained by using a threshold effect in the kinematic hardening, cf. [7] or by using a Ohno-Wang type of model, e.g. cf. [8], [9]. However, due to good results for the later cycles of the experiments the current model is deemed advanced enough for the current purpose. Since the material model used is unable to capture the first loading cycle in combination with an accurate response for later cycles it has been calibrated separately for experiments with $R_{\epsilon}=0$ and $R_{\epsilon}=0.6$ in an in-house code according to a procedure described in [10]. The calibrated material parameter sets are given in Table 1.

The calibrated material model was used to analyse the specimens from the high strain fatigue crack growth tests in a 3D finite element (FE) model in the commercial software Abaqus. Models for three different crack sizes were used $(a=c=1,1.5$ and $2 \mathrm{~mm}$, where $a$ is the crack length and $c$ is half the width of the crack). The crack surfaces are modelled by free nodes. A contact analysis with a rigid surface is applied to prevent crack nodes from penetrating the symmetry crack surface during compression. All FE-analyses are run for 100 loading cycles so that a stable material response is obtained. To enable experiment like loading conditions for the FE-models an explicit displacement

Table 1: Calibrated parameter sets for $R_{\epsilon}=0$ and $R_{\epsilon}=0.6$. The parameters given are $E$, the elastic modulus, $\sigma_{\mathrm{y}}$, the yield stress, $H_{i}$, the kinematic hardening modulus and $B_{\infty, i}$, the saturation value for the kinematic hardening.

\begin{tabular}{|c|c|c|c|c|c|c|c|c|}
\hline$R_{\epsilon}$ & $E[\mathrm{GPa}]$ & $\sigma_{\mathrm{y}}[\mathrm{MPa}]$ & $H_{1}[\mathrm{GPa}]$ & $B_{\infty, 1}[\mathrm{MPa}]$ & $H_{2}[\mathrm{GPa}]$ & $B_{\infty, 2}[\mathrm{MPa}]$ & $H_{3}[\mathrm{GPa}]$ & $B_{\infty, 3}[\mathrm{MPa}]$ \\
\hline 0 & 90 & 359 & 643 & 211 & 14 & 260 & 36 & 86300 \\
0.6 & 89 & 300 & 479 & 264 & 3.73 & 424 & 10.6 & $\infty$ \\
\hline
\end{tabular}



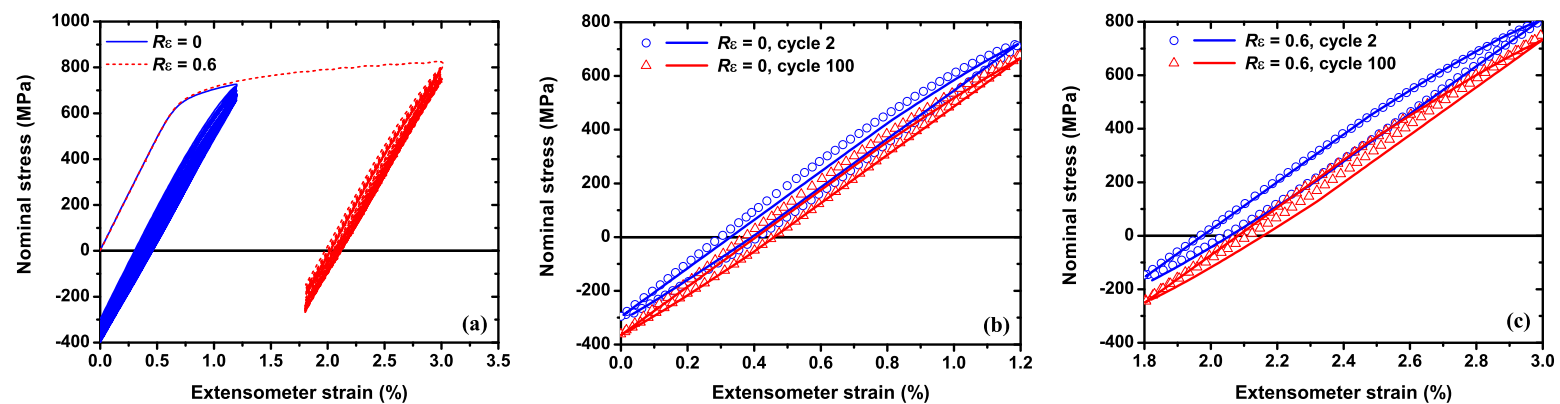

Fig. 1: Stress-strain response for two high strain specimens, one at $R_{\epsilon}=0$ and one for $R_{\epsilon}=0.6$. (a) shows the entire recorded stress-strain response from the tests, whereas (b) and (c) show cycle 2 and 100, respectively, for the two specimens (symbols) and the predicted cycles from the constitutive model (solid lines).

control algorithm, from hereafter referred to as extensometer control, was applied in Abaqus. The cyclic loading of the top nodes are controlled by user subroutine DISP and the displacements at the node corresponding to the placement of the extensometer, i.e. at the middle of the short side of the specimen cross-section $6 \mathrm{~mm}$ from the crack plane, are checked after each time increment by the user subroutine URDFIL, cf. [11].

\section{Results}

Global stress-strain response. The global stress-strain response, in terms of strain measured by the extensometer and nominal stress based on the un-cracked area, for one specimen for each $R_{\epsilon}$-ratio is shown in Fig. 1. It is clear that the specimens undergo extensive plasticity during the first loading excursion. For $R_{\epsilon}=0.6$, the maximum stresses and strains are very high, exceeding $95 \%$ of the monotonic ultimate tensile strength. Individual stress-strain loops corresponding to cycles 2 and 100 are shown in Figs. 1(b) and 1(c). A number of features can be noted from the hysteresis loops:

i The specimens undergo global cyclic plasticity throughout the test. The plastic strain ranges in the relatively stable state are in the order of 0.05 to $0.15 \%$ if calculated as $\Delta \epsilon_{\mathrm{pl}}=\Delta \epsilon-\Delta \sigma / E$, where $\Delta \sigma$ is the measured stress range and $E$ is the Young's modulus, or 0.05 to $0.07 \%$ if measured from hysteresis loop width.

ii The material exhibits a slight cyclic hardening, as seen from the increasing stress amplitude development in Fig. 2(b). From smooth bar LCF tests it can be shown that the material undergoes slow but continuous cyclic hardening (linear with the logarithm of cycle number) at least until around 10000 cycles at a strain range of $1.2 \%$. The apparent softening observed after about 1000 cycles in Fig. 2 is related to the loss of cross-sectional area from the increased crack length rather than a material softening.

iii The mean stress decreases with number of cycles but does not reach zero during the duration of the test. This is reflected in the steadily decreasing, negative values of $R_{\sigma}$ in Fig. 2(c). Again, the rapid decrease towards the end of the test is related to reduction in load carrying area, which affects the maximum stress. The values of $R_{\sigma}$ in the semi-stable state is -0.6 and -0.35 for the tests at $R_{\epsilon}=0$ and 0.6 , respectively.

Figs. 1(b) and (c) also contain the results from the calibration of the constitutive model, showing excellent agreement between measured and predicted loops. The stresses calculated from the reaction forces in the loading direction of the specimen are extracted from FE-analyses for both $R_{\epsilon}$ values and for the three different crack sizes analysed. The stresses from the stable model response of the FE-analyses are compared to experimental stresses in Fig. 2, 3 and 4. The FE-results are shown at the cycle numbers corresponding to the average number of cycles to reach the specific crack lengths in the two duplicate tests. It can be observed that all FE-results compare well to experiments for the shortest crack length of $1 \mathrm{~mm}$, whereas for the larger crack sizes the discrepancy between FE- and experimental results increases. 

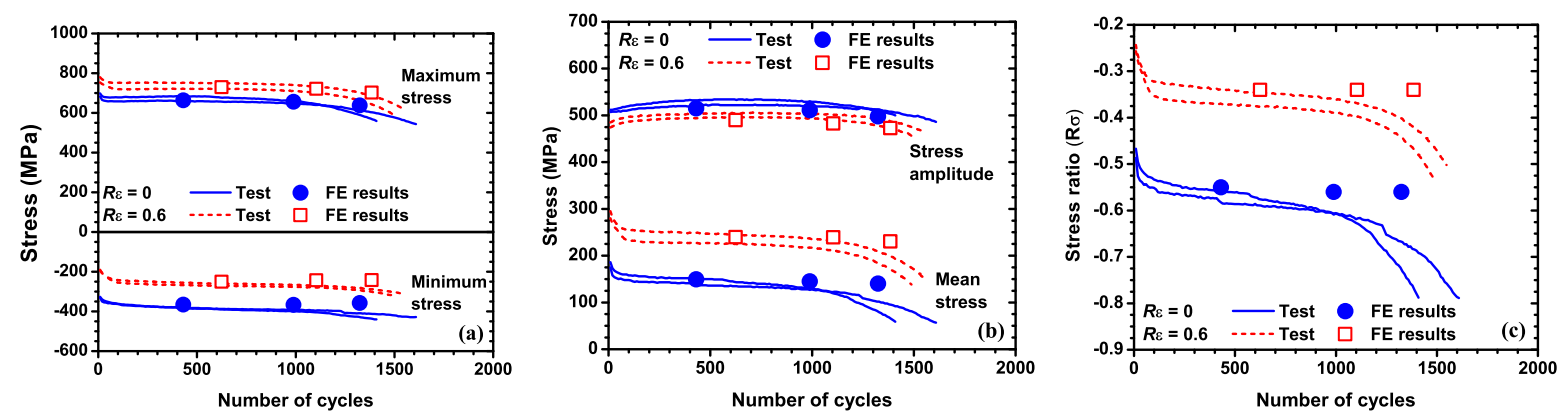

Fig. 2: (a) Development of maximum and minimum stress with cycle number; (b) Development of stress amplitude and mean stress with number of cycles; (c) Development of stress ratio $\left(R_{\sigma}\right)$ with number of cycles.
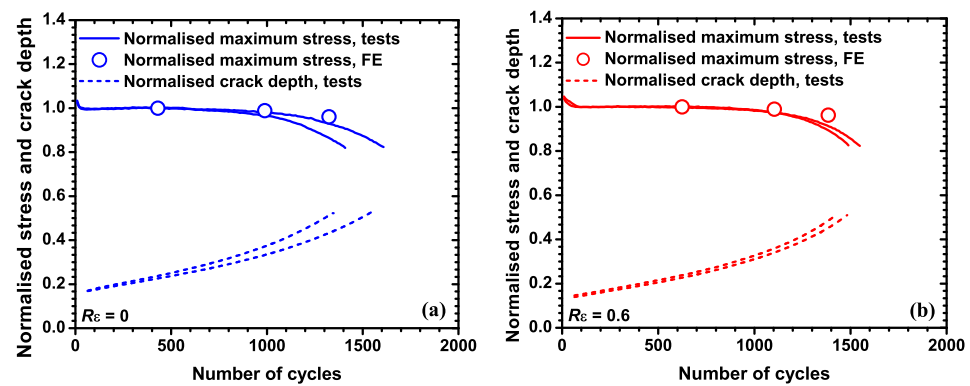

Fig. 3: Crack depth normalised by specimen thickness $(a / B)$ and maximum stress normalised by saturated stress level ( $\left.\sigma_{\max } / \sigma_{\text {sat }}\right)$ vs. number of cycles for (a) $R_{\epsilon}=0$ and (b) $R_{\epsilon}=0.6$.

Relation between crack length and stress response. As the crack grows, the load carrying area in the cracked cross-section is reduced. The effect of crack length on the stress response of the material recorded from the high strain tests can be seen in Fig. 3, where the maximum stress (expressed as normalised stress by dividing with the saturated maximum stress $\sigma_{\text {sat }}$ ) is plotted together with normalised crack length (divided by specimen thickness, $B$ ). It is clear that they are related, especially at the later stages of the test, however the observed drop in maximum load is not captured by the FE-simulations. Fig. 4 shows the correlation between reduction in load carrying area (compared to an un-cracked specimen) and reduction in maximum stress (compared to the saturated stress level $\sigma_{\text {sat }}$ ) for all high strain tests. The results are marked "Un-compensated" in Fig. 4. For short cracks, having an area less than approximately $5 \%$ of the nominal cross-section $(a=1.2 \mathrm{~mm})$, there is essentially no effect of the crack. Above $5 \%$ area reduction the effect of reduced cross-sectional area begins to affect the global stress response and for longer cracks, with an area above $10 \%$ of the nominal area ( $a=1.7 \mathrm{~mm}$ ), a linear relationship is observed. The relation is independent of applied $R_{\epsilon}$ ratio. Again, the deviations between experiments and simulations regarding the drop in maximum stress can be observed. The FE-results, however, compare well to the results from linear elastic analyses run under the same condition (same FE-model in strain control). Upon further examination of the data in Fig. 2(a) it can be seen that there is a slow but continuous decrease of the minimum stress, indicating a continuous shake-down towards the stable $R_{\epsilon}=-1$ situation. The stress amplitude in Fig. 2(b), on the other hand, is stable, indicating that part of the drop in maximum load is a result of the decreasing mean stress rather than crack related area reduction. The experimental load drop can be compensated for this, and the results are shown in Fig. 4, marked as "Mean stress compensated". This data agrees well with the FE-results from both elastic and elastic-plastic analyses, and is still not affected by the applied $R_{\epsilon}$.

\section{Discussion}

In Fig. 5 the measured strains around the modelled specimen, $6 \mathrm{~mm}$ from the symmetry crack surface, are compared for the different crack sizes analysed. The middle of the short specimen face is high- 


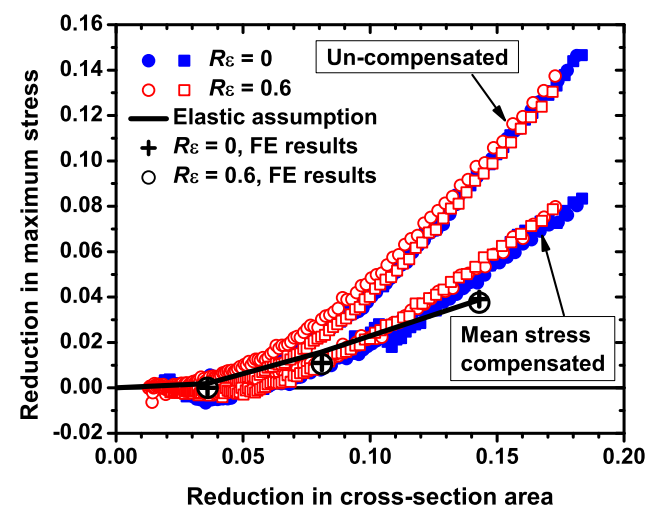

Fig. 4: Relationship between reduction in load carrying area and reduction in maximum stress for all tested high strain specimens. The data marked "mean stress compensated" have been compensated for the mean stress relaxation.
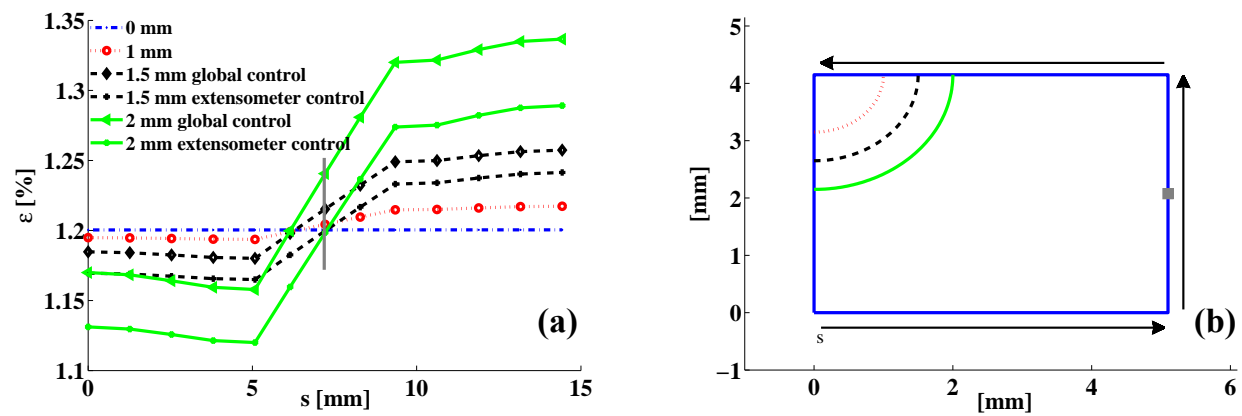

Fig. 5: (a) Strains obtained in FE-analysis during maximum load around the specimen (following coordinate s) $6 \mathrm{~mm}$ from the symmetry crack surface for models with different crack sizes and $R_{\epsilon}=0$. (b) Schematic illustration of the specimen cross-section highlighting the position of the extensometer.

lighted as the aim position of the extensometer in the experimental setup. Results are shown for all crack lengths when the cyclic loading is modelled either by a prescribed displacement of the top nodes giving an effective global strain of $1.2 \%$ (global control), or from the extensometer control algorithm (1.5 and $2 \mathrm{~mm}$ crack lengths only). The results clearly show that the transverse placement of the extensometer will affect the results when the crack is long enough. This could be compared to the case of LCF testing where the extensometer placement with regard to the crack cannot be controlled prior to the experiment, and is of vital importance if simulations are to be used to determine the crack size at a given load drop. The effect will be even more pronounced for round bar specimens as the compliance will be more affected by the crack, which approach a through-crack as it grows. This also shows the benefit of using a simulation based determination of crack length at initiation, as the location of the extensometer can be controlled relative to the crack. If interrupted LCF tests are used to determine the crack length, a large scatter can be expected due to the varying initiation points, whereas worst case scenarios can be determined in simulations to ensure conservatism.

From the results in Fig. 4, it is clear that if the correspondence between load drop and area reduction is to be accurately determined by FE-simulations, rather than interrupted testing, the model must be able to capture the mean stress relaxation occurring for positive $R_{\epsilon}$ ratios. This relaxation is rapid for higher strain ranges, but for typical levels of $\Delta \epsilon$ corresponding to realistic fatigue lives the process is slow and the fully reversed $R_{\sigma}=-1$ state may not be reached. The drawback of this is that the simulations must be run for the same number of cycles as the tests, which for the relevant strain levels may be several tens of thousands of cycles. This is not realistic from a computational cost perspective. Perhaps efficient time integration techniques with extrapolation of load cycles, such as described in [12], could be used. The alternative, which was used here, is to use a model which can accurately predict the stable loop, and compensate the maximum stress obtained from simulations 
using the experimental mean stress relaxation. This was shown to give good results, but require experimental data of sufficient quality. In the present case, the relationship between load drop and area reduction could be well approximated also by a linear elastic FE-analysis using the correct boundary condition in terms of control mode and compensation for the mean stress relaxation.

Another aspect of the mean stress relaxation is that it is rarely accounted for in standard test practices. If the aim is to identify the point of initiation as corresponding to a given relative reduction in stiffness due to the growth of the crack, the simultaneous effect of mean stress relaxation should be treated. If experimentally obtained load drop - crack length correlation curves, such as Fig. 4 are used for strain ranges or $R$-ratios which was not included in the generation of the curves, the inclusion of mean stress effects is crucial to obtain correct values of the crack length.

\section{Conclusions}

- The relationship between reduction of area and drop in maximum load during high strain fatigue crack growth testing is independent of $R_{\epsilon}$, and can be well approximated by linear elastic FEanalyses or elastic-plastic analysis using a Chaboche model and mean stress correction from experiments. The latter is believed to have more general applicability.

- Capturing both maximum/minimum stresses and mean stress relaxation in the constitutive model are vital if simulations are to be used for characterising the crack lengths in LCF tests, without direct experimental data for correction. This is only practically feasible if sufficiently accurate and computational efficient time integration technique (including extrapolation) is available.

- The effect of mean stress correction and extensometer placement is generally ignored in standard LCF testing practice, which can give limited repeatability if interrupted LCF tests are used for determination of crack length.

- When performing FE-simulations for the purpose of determine crack length or load drop, it is important to use a strain control algorithm corresponding to the actual loading conditions and extensometer placement relative to the crack.

\section{References}

[1] K. Bhanu Sankara Rao, M. Valsan, R. Sandhya, S.K. Ray, S.L. Mannan and P. Rodriquez: On the failure condition in strain-controlled low-cycle fatigue, Int J Fatigue, Vol. 7 (1985), p. 141-147

[2] A. Coles, R.E. Johnson and H.G. Popp: Utility of Surface-Flawed Tensile Bars in Cyclic Life Studies, J Eeng Mater-T ASME, Vol. 98 (1976), p. 305-315

[3] ASTM E-647: Standard Test Method for Measurement of Fatigue Crack Growth Rates, Annual Book of ASTM Standards, ASTM, West Conshohocken (2001)

[4] ASTM E-740: Standard Practice for Fracture Testing with Surface-Crack Tension Specimens, Annual Book of ASTM Standards, ASTM, West Conshohocken (2001)

[5] J.L. Chaboche: Constitutive Equations for Cyclic Plasticity and Cyclic Viscoplasticity, Int J Plasticity, Vol. 5 (1989), p. 247-302

[6] S. Bari and T. Hassan: Anatomy of coupled constitutive models for ratchetting simulation, Int J Plasticity, Vol. 16 (2000), p. 381-409

[7] J.L. Chaboche, P. Kanouté and F. Azzouz: Cyclic inelastic constitutive equations and their impact on the fatigue life predicitions, Int J Plasticity, Vol. 35 (2012), p. 44-66

[8] M. Becker and H. Hackenberg: A constitutive model for rate dependent and rate independent inelasticity. Application to IN718, Int J Plasticity, Vol. 27 (2010), p. 596-619

[9] D. Gustafsson, J.J. Moverare, K. Simonsson and S. Sjöström: Modeling of the constitutive behavior of Inconel 718 at intermidiate temperatures, J Eng for Gas Turb Power, Vol. 133 (2011)

[10] R. Brommesson and M. Ekh: Experiments and modelling of the cyclic behaviour of Haynes 282, Technische Mechanik, Vol. 32 (2012), p. 130-145

[11] Abaqus User Subroutines Reference Manual, Version 6.9 (2009)

[12] G. Johansson and M. Ekh: On the modeling of large ratcheting strains with large time increments, Eng Computation, Vol. 24 (2007), p. 221-236 